\title{
JUMPING THE CURVE
}

\author{
René Pellissier ${ }^{1}$ and Paul Kruger \\ Department of Industrial and Systems Engineering \\ University of Pretoria.
}

\begin{abstract}
This paper explores the notion of jumping the curve, following from Handy's S-curve onto a new curve with new rules policies and procedures. It claims that the curve does not generally lie in wait but has to be invented by leadership. The focus of this paper is the identification (mathematically and inferentially) of that point in time, known as the cusp in catastrophe theory, when it is time to change - pro-actively, pre-actively or reactively. These three scenarios are addressed separately and discussed in terms of the relevance of each.
\end{abstract}

\section{KEYWORDS}

Cusp, catastrophe theory, inflection point, change point, saddle point, pro-active, pre-active, reactive transformations

\footnotetext{
${ }^{1}$ René Pellissier is associate professor at the University of South Africa's Graduate School of Business Leadership
} 
Organisations must face and conquer the chaos that exists in the new world order. Today's explosion into telecommunications, science and technology, into global markets, all challenge the old ways of thinking. In this changing environment, the corporate leader cannot assume anymore that his business can resume along the old ways of the past. History teaches that, periodically, society as a whole needs to make a sharp break with old habits. This is also the destiny of business. Business needs to move from chaos, tumult and confusion into clarity - into a new business order. There appears to be neither any half measure nor a piecemeal solution.

Charles Handy's [1] so-called sigmoid (or S-) curve (Figure 1), describes the organisation's natural life cycle. Any new idea, business or industry starts at the bottom of the curve, struggles through early development, increases its expansion, grows and succeeds over time - as represented by the steep gradient of the curve (growth phase). Success gradually slows as market conditions change and new competitors and technologies emerge - as represented by the levelling off of the curve (maturity phase). This is followed by the downward slope of the curve (decline phase).

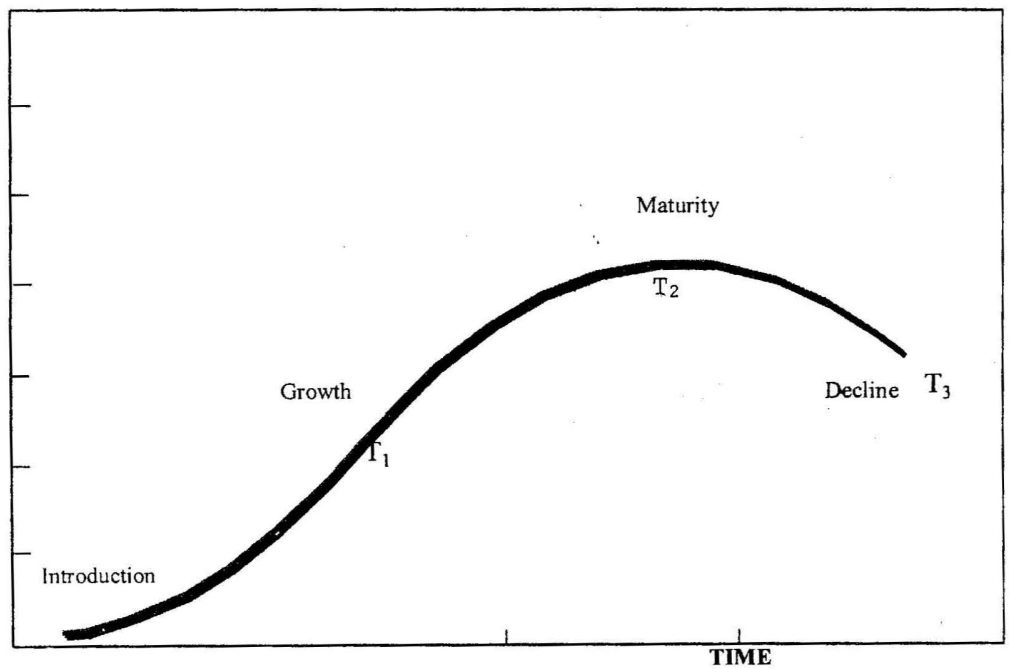

Figure I: Handy's S-curve showing the natural business cycle [1]. 
The situation clearly needs a new boost and organisations need to consider carefully, to anticipate when it is time to jump that curve [2]. It demands of leadership to invent a new curve for their business, discontinuous of the previous one, in order to rise to new, higher levels of success. Every new curve is discontinuous of those preceding it since it is based on a different set of assumptions and business opportunities.

In the following section, the time to change, that point in time denoted by the term the cusp or point of transition, will be explored.

\section{TIME TO CHANGE - IDENTIFICATION OF THE CUSP}

.Hamel and Prahalad [3] believe that organisational transformation is an imperative for every organisation. According to them there are different platforms for the transformation, these being pro-active, pre-active or reactive. A pro-active transformation would reflect acting with foresight (in a calm and considered atmosphere), pre-active transformation would entail planning through the rearview mirror, while reactive transformation generally involves a crisis atmosphere (burning platform). There exists a need for radical change in the growth (proactive) or in the maturity (reactive) phase.

This section addresses the issue of when it is time for radical change, i.e. identifying the specific point in time for the change. In this, it distinguishes between the pro-active, pre-active and reactive transformations ${ }^{2}[16]$.

\subsection{DEFINITION OF THE CUSP}

Formally, the cusp can be defined as that point at which two curves meet [4]. It can be described as the transition or bifurcation point, denoting the passing or change from one place, state or condition to another. Mathematically, the cusp is at the point of inflection of a given curve, while from a statistical viewpoint the cusp is called a change point.

\subsection{MATHEMATICAL DETERMINATION OF THE CUSP}

\footnotetext{
2 Reactive: Tendency to focus on the past; Pre-active: Maintaining the strategic fit by trying to predict the shape of the future and Pro-active: Designing the future and making it happen [16].
} 
Handy's S-curve is often used to describe the life cycle of a product, an idea or organisation. There is a need for radical change in the growth (pro-active), pre-actively in the maturity phase or reactively on the downward slope of the curve. The curve may be described by a number of models, one of which, the Gompertz function, which will be discussed below as an example, after which a model that better suits this, will be proposed. Both will be investigated from a reactive, a pre-active and a pro-active perspective.

In Mathematics, the point of inflection is that point on the curve where the function changes from concave to convex, that is, where $\frac{\delta^{2} S}{\delta T^{2}}$ changes sign. Ideally, the change should be orchestrated at the point of inflection during the growth phase, indicating a pro-active decision to change (that is, designing the future and making it happen). It is also possible to be pre-active in the decision to change (that is, maintaining a strategic fit in terms of our expectation of a possible future), in which case the jump should be orchestrated during the maturity phase at the saddle point. It is also possible (yet not advisable) to be reactive in the decision to change (that is, from a crisis management perspective), in which case the jump will be orchestrated after the maturity phase has been reached on the downward slope of the curve. The latter phenomenon is known as the burning platform scenario.

(a) The Gompertz curve:

The Gompertz curve is generally used to summarise Handy's S-curve. The curve can be summarised by the following function [5]:

$$
\log S=K-M^{T} \text { (this follows from }{ }^{3} S^{\prime}=\log S=\beta_{0}-\beta_{1} \beta_{2}{ }^{T}+\varepsilon \text { ) }
$$

where

$\mathrm{T} \equiv$ time elapsed,

$\mathrm{S} \equiv$ demand for business output, and

$\mathrm{K}, \mathrm{M}$ and $\mathrm{N} \equiv$ constants such that:

$\mathrm{K}$ is known as the upper saturation level, $0<\mathrm{M}<1$ and $\mathrm{N}$ determines how fast the rate of growth declines.

\footnotetext{
${ }^{3}$ For the modified exponential curve, $\beta_{0}=$ the trend value at time zero, $\beta_{1}=$ amount by which the trend value is multiplied to calculate the trend value in the next period $\left(0<\beta^{\prime}<1\right)$, $\beta_{2}$ determines how fast the rate of growth declines (i.e. the closer $\beta_{2}$ is to 0 , the slower the curve will grow toward its upper saturation level) and $\varepsilon=$ the relative movement of the series away from the trend at the time $t$ (error term) [5].
} 
Then three change points (or cusps, $T_{1}, T_{2}$ and $T_{3}$ ) are relevant following from the pro-active, preactive or reactive scenarios discussed earlier. These cusps can be summarised in terms of the first and second derivatives (Table 1).

Table 1: Summary of alternatives for jumping the curve

\begin{tabular}{|c|c|c|c|}
\hline SCENARIO & $\begin{array}{l}\text { CHANGE POINT } \\
\text { (CUSP) }\end{array}$ & $\begin{array}{l}\quad \text { DERIVATIVES }^{4} \\
\text { FIRST } \\
\text { DERIVATIVE } \\
\text { (AROUND T) }\end{array}$ & $\begin{array}{l}\text { SECOND } \\
\text { DERIVATIVE } \\
\text { (AROUND T) }\end{array}$ \\
\hline $\begin{array}{l}\text { Pro-active } \\
\text { (during the growth } \\
\text { phase) }\end{array}$ & $\begin{array}{l}T_{1} \\
\text { (inflection point) }\end{array}$ & $\begin{array}{l}\frac{\delta S}{\delta T}>0 \\
\frac{\delta S}{\delta T}>0\end{array}$ & $\begin{array}{l}\frac{\delta^{2} S}{\delta T^{2}}>0 \\
\frac{\delta^{2} S}{\delta T^{2}}<0\end{array}$ \\
\hline $\begin{array}{l}\text { Pre-active } \\
\text { (during the maturity } \\
\text { phase) }\end{array}$ & $\begin{array}{l}\mathrm{T}_{2} \\
\text { (saddle point) }\end{array}$ & $\begin{array}{l}\frac{\delta S}{\delta T}>0 \\
\frac{\delta S}{\delta T}=0 \\
\frac{\delta S}{\delta T}<0\end{array}$ & $\begin{array}{l}\frac{\delta^{2} S}{\delta T^{2}}<0 \\
\frac{\delta^{2} S}{\delta T^{2}}<0 \\
\frac{\delta^{2} S}{\delta T^{2}}<0\end{array}$ \\
\hline $\begin{array}{l}\text { Reactive } \\
\text { (during the decline } \\
\text { phase) }\end{array}$ & $\begin{array}{l}\mathrm{T}_{3} \\
\text { (not an inflection point if } \\
\text { it is accepted that the } \\
\text { curve will asymptotically } \\
\text { reach } 0 \text { ) }\end{array}$ & $\begin{array}{l}\frac{\delta S}{\delta T}<0 \\
\frac{\delta S}{\delta T}<0\end{array}$ & $\begin{array}{l}\frac{\delta^{2} S}{\delta T^{2}}<0 \\
\frac{\delta^{2} S}{\delta T^{2}}<0\end{array}$ \\
\hline
\end{tabular}

(i) Pro-active scenario:

It follows that the point in time (say $T_{1}$ ) can be obtained by solving $\mathrm{T}$ from the equation $\frac{\delta^{2} S}{\delta T^{2}}=$

0 . Solving $T_{1}$ from the equation, $\frac{\delta^{2} S}{\delta T^{2}}=0$, one obtains

${ }^{4}$ For the Gompertz curve cited above, $\frac{\delta S}{\delta \mathrm{T}}=-\mathrm{MN}^{\mathrm{T}} \log \mathrm{N}\left(\mathrm{K}-\mathrm{MN}^{\mathrm{T}}\right) \exp \left(\mathrm{K}-\mathrm{MN}^{\mathrm{T}}\right)$ and

$$
\frac{\delta^{2} S}{\delta T^{2}}=-M \log N\left[\log N \cdot N^{T}\left(K-M N^{T}\right)-N^{T}\left(M \log N \cdot N^{T}\right)\right] .
$$


$\mathrm{T}_{1}=\log _{\mathrm{N}}\left(\frac{\mathrm{K}-\mathrm{M}}{\mathrm{N}}\right)$, with $\mathrm{K}>\mathrm{M}(0<\mathrm{M}<1)$ and $\mathrm{N}$ positive.

(ii) Pre-active scenario:

It follows that the point in time (say $\mathrm{T}_{2}$ ) can be obtained by solving $\mathrm{T}$ from

$\frac{\delta S}{\delta T}=M N^{\mathrm{T}} \log \mathrm{N}\left(\mathrm{K}+\mathrm{MN}^{\mathrm{T}}\right)=0$. Thus, it follows that

$\mathrm{MN}^{\mathrm{T}} \log \mathrm{N}=0$, thus $\mathrm{T}_{\text {cusp }}=-\infty$ or $\mathrm{K}-\mathrm{MN}^{\mathrm{T}}=0$, thus $\mathrm{T}_{2}=\frac{\log \frac{\mathrm{K}}{\mathrm{M}}}{\log \mathrm{N}}=\log _{\mathrm{N}} \frac{\mathrm{K}}{\mathrm{M}}$,

with $\mathrm{K}>\mathrm{M}(0<\mathrm{M}<1)$ and $\mathrm{M}$ positive.

(iii) Reactive scenario:

Since there is no inflection point because of the assumption that the function asymptotically tends to infinity, no unique solution exists under this scenario. This suggests that it is not possible to be reactive in decision-making if the Gompertz curve is assumed relevant.

Ideally, one would expect the jump to occur around the $T_{i}$ value $(i=1,2)$ obtained under the proactive or pre-active scenarios. Any movement after the saddle point has been reached $\left(\frac{\delta S}{\delta T}=0\right)$ is reactive in nature, the optimum solution being around the point of inflection. The jump cannot be orchestrated under a reactive scenario since there does not exist a solution. The following general family of functions is proposed as they follow Handy's suggested shape, but provide cleaner solutions under the scenarios discussed above.

(b) Proposed model for the pro-active, pre-active or reactive scenarios:

The following family of functions provide greater scope in terms of applicability and form for Handy's S-curve. 
Let $f(t)=-b(c t-a) \exp (c t-a), \quad 0 \leq t \leq \frac{a}{c} ; \quad$ where $a, b$ and $c$ are positive constants with respect to the specific business or industry and $t$ is the expected point in time to jump the curve ${ }^{5}$. (Note that it is also possible to add a constant term to the model with no loss of generality.)

As before, three cusps are relevant pertaining to the different scenarios. These lead to different signs of the first and second derivative, as in Table 1. Letting $\frac{\mathrm{d}^{2} \mathrm{f}}{\mathrm{dt}^{2}}=0$, one finds $\mathrm{T}_{1}=\frac{\mathrm{a}}{\mathrm{c}}$ resulting in a negative second derivative. From this, one may conclude that the pro-active cusp point in time occurs after the inflection point. Letting $\frac{\mathrm{df}}{\mathrm{dt}}=0$, one finds $\mathrm{T}_{2}=\frac{\mathrm{a}+1}{\mathrm{c}}$ resulting in a negative first derivative. From this, it follows that the pre-active cusp point in time occurs after the saddle point (where $\frac{\mathrm{df}}{\mathrm{dt}}$ is negative). As before, no unique solution exists under the reactive scenario because of the assumption of an asymptotic distribution.

In conclusion, the main problem with the above methodology, is that the specific functional relationship should be known a priori, with $\mathrm{T}$ the only independent variable. The above method would serve organisations that optimally have large enough data sets (generally, time series data) available to know a priori the behaviour (distribution) of the variable(s) they are studying (for example, sales data) and need to make predictions in terms of new product development and the obsolescence of existing products (the second proposition serves a wider variety of distributions than the first). Whether these values are in fact readily and abundantly available, is questionable - one reason being that time series analysis requires typically large data sets (rare in modern industries) to be accurate in their predictions. The Gompertz curve, since it follows Handy's Scurve could be used if not enough data is available to determine a model specific to the organisation's own history or industry. Alternatively models of the form proposed under (ii) could be fitted using statistical modelling techniques.

\subsection{INFERENTIAL DETERMINATION OF THE CUSP}

$5 \frac{d f}{d t}=-b c \exp (c t-a)[1-c t+a]$ and $\frac{d^{2} f}{{d t^{2}}^{2}}=-b c^{2} \exp (c t-a)(a-c t)$ 
In Statistics, the term change point model is generally taken to denote those models in which a change in parameters or functional form occurs at some point in a sequence of observations [6].

A description of the simplest type of change point problem is given by Lombard [7]. Consider a time ordered sequence of independent observations $\mathrm{x}_{1}, \mathrm{x}_{2}, \ldots, \mathrm{x}_{\mathrm{n}}$ with distribution functions $\mathrm{F}_{1}, \mathrm{~F}_{2}$, ..,$F_{n}$. If $F_{1}=F_{2}=. .=F_{n}$, the data are homogeneous, that is $x_{1}, x_{2}, . ., x_{n}$ constitute a random sample from a fixed distribution. More generally, suppose that, for some integer $\tau(1 \leq \tau<n)$, $x_{1}$, $\mathrm{x}_{2}, . ., \mathrm{x}_{\mathrm{n}}$ have a common cumulative distribution function $\mathrm{F}$, while $\mathrm{x}_{\tau+1}, \mathrm{x}_{\tau+2}, \ldots, \mathrm{x}_{\tau^{+} \mathrm{n}}$ have cumulative distribution function $G$. Then a change of distribution has occurred with $\tau$ called the change point.

\section{PRINCIPLES OF HYPOTHESIS TESTING FOR CHANGE POINT MODELS}

(i) General hypotheses:

For the above-mentioned case, the following is valid:

Test $\mathrm{H}_{0}: \mathrm{F}=\mathrm{G}$ (hence $\mathrm{F}_{1}=\mathrm{F}_{2}=. .=\mathrm{F}_{\mathrm{n}}$ ), against $\mathrm{H}_{\mathrm{a}}: \mathrm{F} \neq \mathrm{G}$, and subsequently, estimate $\tau$ in the event of rejection of $\mathrm{H}_{0}$.

If $\tau$ is known, this becomes a two sample problem for which various parametric and non parametric tests exist. The distinguishing factor in change point analysis, is that $\tau$ is not known a priori.

(ii) General construction of test statistics:

Construction of tests for $\mathrm{H}_{0}$ can be based on the well-known two-sample tests. Consider $Z_{\tau}$ an appropriate test statistic for the two sample problem involving $x_{1}, x_{2}, \ldots, x_{\tau}$ and $x_{\tau+1}$, .. , $\mathrm{x}_{\mathrm{n}}$. Then obvious choices of test statistics for testing $\mathrm{H}_{0}$ against an alternative involving an unknown change point are $\max _{1 \leq r<n} Z_{r}$ or $\frac{1}{n} \sum_{1 \leq r<n} Z_{r}$.

The main focus in statistical literature involves the derivation of exact or approximate distributions of such test statistics. Examples of these are discussed below.

(iii) Parametric tests:

Parametric tests involve tests where the underlying distribution (normal, exponential, or otherwise) may be assumed known. Let $x_{1}, x_{2}, . ., x_{n}$ be independent and normally 
distributed with respective means $\mu_{1}, \mu_{2}, ., \mu_{n}$ and common variance $\sigma^{2}$. The simplest model for change in mean after the $r^{\text {th }}$ observation $(1 \leq r<n)$ is given by $H_{0}: \mu_{i}=\mu^{*}$, against $\mathrm{H}_{\mathrm{a}}: \mu_{\mathrm{i}}=\mu \mathrm{I}(1 \leq \mathrm{i}<\tau)+\mu^{*} \mathrm{I}(\tau+1 \leq \mathrm{i}<\mathrm{n})$ for some unspecified value $\tau$ and indicator function $\mathrm{I}$. The subsequent test statistic is $U_{\mathrm{n}}=\max (|\mathrm{T} \tau|: 1 \leq \tau<\mathrm{n})$, which follows the $t$ distribution with $\mathrm{n}-2$ degrees of freedom. Also, $\mathrm{T} \tau$ corresponds to the familiar test statistic used for a two sample test (independent observations) of the means, the latter respectively being $\overline{x_{\tau}}$ and $\overline{x_{\tau}^{*}}$ for the two groups and pooled variance $\sigma^{2}$. The major problem in this, is that the two groups need not necessarily be independent.

Tests also exist for the exponential distribution, the binomial distribution and the Poisson distribution.

(iv) Situations that may complicate these derivations [7]:

The above methodology assumes some underlying distribution - the knowledge of which, a priori, is generally not available. Moreover, the number of change points are assumed to be at most one (known as AMOC). (The latter may be corrected using procedures based on ranks.) Another problem is the presence of nuisance parameters, that is, to test for a change in the variance of a normal distribution with fixed but unknown mean. One should also determine whether some value can be classified as a significant change point or a statistical outlier. (Simple rank tests may solve this problem.) Lastly, the $x_{i}$ are not independent, but are generated by some auto-regressive scheme - thus negating the assumption of independence in the $t$ test above.

\section{(v) Rank tests:}

Suppose that $\mathrm{x}_{1}, \mathrm{x}_{2}, . ., \mathrm{x}_{\mathrm{n}}$ are independent with unspecified density functions, given by $\mathrm{f}_{1}{ }^{\theta}, \mathrm{f}_{2}{ }^{\theta}, \ldots, \mathrm{f}_{\mathrm{n}}{ }^{\theta}$. and let $\mathrm{r}_{\mathrm{i}}$ denote the rank of $\mathrm{x}_{\mathrm{i}}, 1 \leq \mathrm{i}<\mathrm{n}$. The distribution of the rank vector $r_{i}$ does not depend upon the underlying density provided that the $\theta$ s are all the same. The simplest way of constructing a non-parametric test is to replace $x_{j}$ in the parametric procedure by $\varphi\left\{\frac{\mathrm{r}_{\mathrm{i}}}{(\mathrm{n}+1)}\right\}$, where $\varphi$ is an appropriate score function defined and integrable on the interval $(0 ; 1)$, depending upon the type of parameter involved (location or scale). 
In the event that the null hypothesis of no change point is rejected, one has to determine the change point. An obvious estimate of $\tau$ is the point $\vec{\tau}$ at which the absolute standardised cusum $\frac{\left|\mathrm{C}_{\mathrm{k}}\right|}{\sqrt{\mathrm{k}(\mathrm{n}-\mathrm{k}) / \mathrm{n}}}$ attains its maximum, where $\mathrm{C}_{\mathrm{k}}=\sum_{i=1}^{n}\left(\mathrm{x}_{\mathrm{i}}-\overline{\mathrm{x}_{\mathrm{n}}}\right), 1 \leq \mathrm{k}<$ n. This estimator $\bar{\tau}$, is not sufficient for the parameter (Hinkley, 1970) $\tau$ since the distribution of the sample $x_{1}, x_{2}, . ., x_{n} \mid \vec{\tau}$ is not independent of $\tau$. Moreover, it is not possible to obtain a consistent estimator for $\tau$, because increasing the sample size has no effect on the shape of the likelihood function. Thus, especially in the non-parametric case, no satisfactory estimation of the change point is yet available.

\section{(vii) Regression analysis:}

Suppose that $x_{1}, x_{2}, . ., x_{n}$ have the linear model structure $x_{i}=A_{i} \beta_{i}+e_{i}$, where

$A_{i}=1 \times p$ row vector of known constants,

$\beta_{\mathrm{i}}=\mathrm{px} 1$ column vector of unknown regression constants and

$\mathrm{e}_{\mathrm{i}}=$ independent and identically distributed random variables.

Then the simplest regression change point model tests $H_{0}: \beta=\beta^{*}$, against $H_{a}: \beta_{i}=\beta$ for i $\leq \tau$, and $\beta_{i}=\beta^{*}$ for $i>\tau$.

Much research has been done in this field, although few offer exact or asymptotic tests. One aspect that has received attention, is the situation where $\mathrm{x}_{1}, \mathrm{x}_{2}, \ldots, \mathrm{x}_{\tau}$ and $\mathrm{x}_{\tau+1}, \ldots, \mathrm{x}_{\mathrm{n}}$ form parts of two time series with spectral densities $f_{1}$ and $f_{2}$ respectively. Software has been developed by Venter and Steele [9] locally, called ABRUPT CHANGE POINT ANALYSIS (ACPA), written in visual basic, that determines, for a given data set, the possible change points - not necessarily AMOC. The programme assumes independence of observations.

In most business applications, data are time-ordered, that is, $x_{i+1}$ is observed after $x_{i}$ and constitute a time series. Nevertheless, the full data set, $\mathrm{x}_{1}, \mathrm{x}_{2}, \ldots, \mathrm{x}_{\mathrm{T}}$ is available for analysis and the situation differs from that encountered in sequential data analysis. In the latter case, the data are analysed with the advent of every new observation, the objective being to detect a change as soon as possible at the onset. The sample size is generally not determined by a data dependent 
stopping rule and the analysis is retrospective by nature. Lastly, the methodology depends to a large extent on known probability distributions - generally not easily determined empirically.

The above methods requires a priori determination from the organisation to determine the closest fit with one of the above (or some other) function that summarises their past behaviours.

The authors partially disagree with Macmillan [10] who states that it is still possible for organisations to jump the curve during the decline phase. From the definitions above, this strategy is deemed neither proactive, nor pre-active and, although arguably, in use, not appropriate as it cannot be termed strategy.

\section{CATASTROPHE THEORY}

The mathematics of smooth, continuous change cannot explain abrupt or sudden changes. Models which result from the use of such mathematics, tend to imply that sudden change is not permissible, or at least, not reasonable. However, sudden changes do occur. Catastrophe theory is concerned with the phenomena of rapid or sudden change. The French mathematician, René Thom, proposed and developed these theories to explain the behaviour of systems. In 1972, he postulated that there were elementary catastrophes represented by mathematical equations that described generalisations of systems that move rapidly from one state to another. It helps to model discontinuous, abrupt changes in a variable (behaviour) as a result of small, continuous changes on one or more other (control) variables.

\subsection{DEFINITION}

According to Karathanos, Pettypool and Troutt [11], catastrophe theory allows for models for which abrupt change is not only permissible but expected. Instead of experiencing the surprise of unexpected movement or behaviour, one may expect catastrophe theory to help explain (and possibly prepare for) the sudden changes in the behaviour variable. Moreover, Wright [12] believes the theory useful in handling situations in which qualitative data are prevalent and in which qualitative management decisions have to be made. The theory may also assist in the determination of appropriate data for quantitative decision-making.

\subsection{CUSP CATASTROPHE MODELS}


The advantages of using cusp catastrophe models (response surfaces) are twofold [13]:

$>$ Their ability to capture in a single response surface both incremental and sudden shifts in strategic response, and

$>$ There exist means for estimating such models containing multivariate constructs that are necessary for dealing with complex strategy variables.

In this, the name may be misleading since it infers only catastrophic situations (earthquakes, tornados or floods), whilst, in reality, the term refers to sudden discontinuous behavioural shifts in the response system. Catastrophes may be described as points whose fundamental processes move towards states of minimum or maximum potential (for example, a clock pendulum stopping). Such models are only appropriate for so-called state-descriptive systems. In these systems, the current state depends upon the prior state, and, statistically, would show high levels of autocorrelation in a regression analysis. In catastrophe theory, the state variable exhibits the rapid change and the change is caused by control variables. Thus, there is some correlation between the 'state' and the 'conditions' the system finds itself under. Where this is not the case, catastrophe theory is inappropriate.

Scapens, Ryan and Fletcher [14] define the cusp catastrophe theory as ' .. concerned with the analytical explanation of sudden changes in the behaviour of a system, arising as a result of smooth changes in the factors which determine the attainable equilibrium states of that system.' The state in which one finds something, is a function of the conditions in which it is embedded. For example, water is frozen below zero degrees Celsius at atmospheric pressures, whilst water boils if the temperature reaches one hundred degrees. Hence, for a given temperature, a physicist is able to determine the state in which water in its liquid form would be found. At a social level, Governments try to take actions and create conditions whereby the population will continue to vote for them, or organisations try to create conditions (wages, benefits and others) that will avoid the state of labour unrest. Indeed, a significant proportion of the quality philosophy, is based on the premise that, under the right conditions (for instance, empowerment of employees), the right state will be achieved (for instance, quality products). Organisations try various techniques to alter the conditions, for example: cross-functional teams, delayering, training and management by objectives. 
According to catastrophe theory, not all systems follow the natural law that there is a direct correlation between the state of the system and the current operating conditions, and, in some situations, under apparently identical conditions two states are possible. There is some sort of jump from the one state to the other. This jump is typical of catastrophic systems and may occur without being noticed if no mechanism is in place to detect this. This results in the possibility of overcompensation. Typically, in organisations, there may be a drive towards decentralisation or reengineering with every unit and element in the system driving towards that goal. The result may be fragmentation, uncoordinated efforts negating the drive towards success. Hence, long before the optimum position of balance has been attained, the conditions are already in place and the system goes too far the other way.

\subsection{MATHEMATICAL IDENTIFICATION OF THE CUSP}

Gresov, Haveman and Oliva [13] cite the following generic form of the cusp function:

$$
f(z)=1 / 4 z^{4}-x y-1 / 2 y z^{2} \text {. }
$$

Thus, mathematically, the cusp model is given by the set of maxima (or minima) points

$$
\frac{\partial f}{\partial z}=z^{3}-y z=0
$$

The following holds for the above variables:

$\mathrm{X}, \mathrm{Y}=$ independent variables,

$\mathrm{Z}=$ dependent variable,

$\mathrm{X}, \mathrm{Y}$ and $\mathrm{Z}$ are assumed latent constructs that can be estimated,

$\mathrm{X}=$ normal factor because $\mathrm{Z}$ changes directly with $\mathrm{X}$,

$\mathrm{Y}=$ splitting factor because the response surface bifurcates as the value of the variable is increased beyond some point known as the singularity.

Additional knowledge of the system is gained from the slope of the tangent to the surface at any point. A change in the magnitude of the slope indicates the approach of discontinuity. As before, the second (partial) derivative provides information on the behaviour of the system: $\frac{\partial^{2} f}{d z^{2}}=3 z^{2}-$ $\mathrm{y}$, from which the moment of inertia (cusp or change point) may be derived as $3 z^{2}-\mathrm{y}=0$, namely $z=\sqrt{\frac{y}{3}}$. Note that this approach differs from that presented by Gresov, Haveman and 
Oliva [13] but these authors are satisfied that the above is mathematically solid and fits the structure presented in previous section.

\section{BUSINESS APPLICATION OF THE CUSP}

'On the cusp' in a business sense, refers to the era of discontent between the production orientation of the past and the current Information Age. Thus, the cusp could also refer to the movement towards the Information Age or onto a different epoch. On a smaller scale, organisations need to determine when they are on the cusp of their current business and be able to jump onto a new curve to bring them competitive advantage.

An application of the cusp catastrophic model is presented by Scapens, Ryan and Fletcher [14]. They provide an example in which credit extension is a function of return on funds and the organisation's operating risk. The apparent continuous relationship between credit extension and return on funds will change once the organisation's operating risk is taken into account. The net effect of the addition of the variable (operating risk) in the model is that, while a small variation in return by a successful organisation may not change the credit worthiness class to which the organisation is assigned, a change in return that exceeds some limit will cause a substantial jump in the relationship between credit extension and return on funds (also operating risk). This may be represented by a catastrophic manifold which embodies one state variable and two control variables, the normal factor and the splitting factor which is responsible for the jump conditions in the model. Although there is a jump in the state variable, both the other variables are smooth. The jump in the state variable has not resulted from an assumption of the existence of a jump in either of the control variables. The model is appropriate because the causes of the jump is endogenous, whereas normal plotting of the state variable against each of the control variables results in a fruitless search for dummy variables or any other exogenous variable in order to explain the irregular pattern in the data.

It is also possible to statistically determine the cusp. The procedure involves multivariate modelling known as GEMCAT [15]. The procedure is computer-based and is described in full in this article.

Clearly it is important to have some notion of whether any jump is likely for the organisation (or business or idea). Most writers focus on the tendency of organisations to execute the jump while 
on the downward slope of the curve. The authors contend in this contribution, that it is precisely this tendency that causes the failure of organisations in executing a successful transition from the old to the new.

From an organisational perspective, leaders should continually question the conditions and states of their systems. These may include questions like:

$>$ What are the benefits the organisation is trying to realise?

$>$ Are the actions taken realising the planned objectives or are they becoming an end in themselves?

$>$ Does management have a clear vision as to where and when the reorganisation is intended to stop?

What is good about the current state and conditions and how may these be preserved?

At a higher level, three important drivers behind the organisational structure are [2]:

$>$ information flows,

$>$ the need for policy consistency, and

$>$ speed of reaction.

Changes in these variables may lead to a change in the relationship between the center and the periphery of an organisation. Most systems (business or governmental) are still tied to the almost universal principal that all members should report to a single point of line responsibility. This is contrary to emerging organisational structures with more natural structures through technology. Imperato and Harari [2] suggest that, reduction of single point responsibilities (and the resultant waste of resources and energy) produces a more stable, yet flexible structure that could be allowed to adapt steadily to a changing environment rather than by a series of dislocating quantum jumps.

\section{MANIFESTING THE CHANGE}

The jump discussed above, can be successfully orchestrated if any of the following four principles (or cultures) are adhered to [2]:

$>$ an innovative culture,

$>$ a culture of coherence,

$>$ an organisational culture of putting the customer first, and, most importantly, 
a culture of reengineering technology.

These are expanded upon below.

(i) An innovative culture: Successful organizations focus continually on tomorrow's markets. They encourage risk and creativity in order to change the status quo and create ' new'. It looks ' ...a customer ahead' [2] by continually preparing itself for obsolescence.

(ii) A culture of coherence: An organization that jumps the curve needs an instrument to bind it together. It needs shared values in an order where diversity will in fact induce creativity and innovation. It is important that leaders reward those that support the new organisational values and priorities.

(iii) An organisational culture of putting the customer first: Commitment to the customer must be genuine and shared. Tomorrow's leader has to bring a spirit of responsibility to replace expedient and self-serving attitudes. Customers form the primary stakeholder group, employees form the second stakeholder group, and the organization should regularly assess attitudes and satisfaction levels and perceptions for all stakeholder groups.

(iv) A culture of reengineering technology: Jumping the curve means building the organisation around technology in order to increase productivity in a leader-led commitment to day to day organisational renewal.

Creating change needs to address a new philosophy that better fits the strategic intent and organisational structure, possibly including significant training, education and structures. These should focus on creating an organisational environment (or culture) in which people's behaviour and performance are aligned with the values and ideals envisioned by the company. Putting such a system into place may take months or years, since creation is often easier than transformation.

\section{SECOND CURVE ORGANISATIONS}

Moving from the machine metaphor for an organisation, Imperato and Harari [2] contend that organisations have to become intelligent. Information flows and associated processes have to be used as organising principle. Thus, they contend that an organisation may expect dramatic improvements in building itself and its software around the customer. This aspect involves more 
than a ' design for technology' -mediated organisational structure. It acknowledges that there is replacement of the old ways of doing business. From organisation as machine, the metaphor moves to continual innovation, flexibility and speed. Thus, the new organisation must efficiently tie together interconnectivity, permeable boundaries and the importance of intangibles. Thus, second curve organisations have successfully and effectively negotiated the transition from mass (customisation) to brain and intelligence. Second curve organisations are leaner and more flexible, while enlarging their intelligence and knowledge base. There may be limited numbers of people, but unlimited information and intelligence. Following on the self-organising theory of chaos theory, leaders should [2]: '.. build the organisation around the software and the software around the customer'. This entails far more than integrating technology. It also refers to the organisation's processes and systems to enhance responsiveness. It entails building the organisation around the software, means organising production and work around knowledge, whereas building the software around the customer effectively puts the customer as central focus. The above self-organising principle intends to make the organisation into an intangible knowledge reservoir that holds the collective knowledge and intelligence of all its members. The capacity of renewal, is seen as a function of intelligence and openness, whereby the organisation should continually learn.

The transition from mass to brain described above makes aggressive use of IT in four distinct initiatives. These are:

Leverage knowledge across the organisation.

Accelerate the development of collaborative work within the organisation and between organisations.

$>$ Prioritise efforts that lead to mass customisation, slender marketing and individualised customer sets.

$>$ Liberate the people from the constraints of the paper dependent environment.

\section{CONCLUSION}

The shift from the Industrial to the subsequent Information age has significantly altered the nature of the workplace, the worker and, thence, the work. Industrial age workers were located primarily in urban factories where they engaged in routine work, often on an assembly line. They worked a specific shift, punched a time clock and performed tasks under close supervision. A good worker was one who was reliable and passive, capable of modest dexterity. 
In contrast, due to modern technology, Information age workers can be located anywhere and can conduct much of their work anytime. For example, a telecommuter can be at home for children after school and make up for such time during the evening. The prized worker is one who learns quickly and continuously, who works collaboratively and who is comfortable in an environment of risk and experimentation. The new knowledge workers perform their work generally without supervision. Those who are engaged in collaborative efforts do so as members of self managed teams.

No organisation can escape the need to re-skill its people, redesign its processes, reshape its product portfolio or redirect its resources. Jumping the curve means conforming to a new set of values, theories and practices. It obviously involves a change in corporate culture and technology influences It means changing ones way of thinking, from doing things right (efficiency) to doing the right thing (effectiveness). It means switching from internal thinking to external thinking. More than that, generally that curve has to be invented - it is lying in wait to be discovered. The above served as guideline to leaders on when it is time to reinvent their businesses. Doing so is far more challenging.

\section{REFERENCES}

[1] Handy, C. 1995. The Empty raincoat. London: Arrow books.

[2] Imperato, $\mathrm{N}$ and Harari, O. 1994. Jumping the Curve. San Francisco: Jossey-Bass Publishers.

[3] Hamel, G. and Prahalad, C.K. 1994. Competing for the future : Breakthrough strategies for control of your industry and creating markets of tomorrow. Boston Mass: Harvard Business School Press.

[4] The Oxford Dictionary for the Business World. Oxford: Oxford University Press. 1993.

[5] Farnum, N.R. and Stanton, L.W. 1993. Quantitative forecasting methods, Boston, PWSKent Publishing Company.

[6] Mariott, F.A.C. 1990. Dictionary of statistical terms. $5^{\text {th }}$ edition. New York: Wiley.

[7] Lombard, F. 1989. Some recent developments in the analysis of change point data. South African Statistical Journal. 23: 1-21.

[8] Hinkley, D. 1987. Inference about the change point in a sequence of random variables. Biometrika, 57: 85-93. 
[9] Venter, L and Steele, S.J. 1995. Abrupt change point analysis software, University of Potchefstroom.

[10] Macmillan, I.C. 1988. Controlling competitive dynamics by taking strategic initiative. The academy of management EXECUTIVE, Vol II, no 2 pp 111-118.

[11] Karathanos, P, Pettypool, M.D and Troutt, MD. 1994. Sudden lost meaning: A catastrophe?. Management Decision, 32(1): 15-19.

[12] Wright, D.J. 1993. Catastrophe theory in management and decision making. Journal of the operational research society, 34(10).

[13] Gresov, C, Haveman, H.A. and Oliva, T.A. 1993. Organisational Design, inertia and the dynamics of competitive response. Organization Science, May, 4(2): 181-208.

[14] Scapens, R. W. Ryan, R.W. and Fletcher, 1. 1981. Explaining corporate failure: A catastrophe theory approach. Journal of business finance and accounting, 8, Spring, 1-26. [15] Oliva, T.A., Desarbo, W.S., Day, D.L. and Jedid, K. 1987. GEMCAT: A general multivariate methodology for estimating catastrophe models. Organisational Science, Sept 4(3), 120-124.

[16] Ackoff, R. 1981. Creating the corporate future. New York: John Wiley. 